\title{
Globe
}

Revue internationale d'études québécoises

\section{Roch Côté [éd.] : Québec 2002. Annuaire politique, social, économique et culturel. Montréal, Fides, 2001}

\section{Jean-Michel Lacroix}

Volume 6, numéro 2, 2003

URI : https://id.erudit.org/iderudit/1000821ar

DOI : https://doi.org/10.7202/1000821ar

Aller au sommaire du numéro

\section{Éditeur(s)}

Globe, Revue internationale d'études québécoises

ISSN

1481-5869 (imprimé)

1923-8231 (numérique)

Découvrir la revue

Citer ce compte rendu

Lacroix, J.-M. (2003). Compte rendu de [Roch Côté [éd.] : Québec 2002. Annuaire politique, social, économique et culturel. Montréal, Fides, 2001]. Globe, 6(2),

175-176. https://doi.org/10.7202/1000821ar d'utilisation que vous pouvez consulter en ligne.

https://apropos.erudit.org/fr/usagers/politique-dutilisation/ 


\section{Recensions}

\section{Roch Côté [éd.]}

Québec 2002. Annuaire politique, social, économique et culturel.

Montréal, Fides, 2001.

Ce volume élégant, maniable, bien relié, a la facture d'un dictionnaire ou d'un ouvrage de référence devenu incontournable; il est attendu chaque année avec tant d'impatience depuis 1996 que cette septième livraison a devancé notre attente en étant publiée dès le troisième trimestre de 2001. Par comparaison avec l'édition de 2001, son volume a augmenté en passant de 529 à 718 pages. On retrouve avec plaisir le graphisme de Gianni Caccia, et les textes sont produits par une équipe de 70 collaborateurs dont certains assurent une continuité tout en s'ouvrant à de nouveaux apports. Parmi les nouveautés, on aura remarqué avec satisfaction une chronologie des événements marquants de l'année, sous la plume de Pascal Faucheur. Pour ce qui est de la continuité, on retrouve un groupe d'articles sur le territoire québécois, auquel s'ajoute de façon curieusement placée un bilan des activités du CIEQ (Centre interuniversitaire d'études québécoises, p. 392 et suivantes).

L'ordre de présentation des articles a changé : la rubrique concernant l'économie vient plus tôt tandis que la culture est reléguée à la fin ; cette dernière s'avère toutefois plus étoffée, car elle inclut des développements sur l'éducation et sur les médias. On relève un titre dont l'ambiguïté est intéressante, puisqu'il est question de l'état du Québec (en attendant l'État du Québec, sans doute appelé de leurs vœux par un grand nombre de collaborateurs dont on sent l'enthousiasme partisan pour la cause québécoise). On a apprécié la mise en relief particulière des questions de population, si vitales pour l'avenir de la Belle province, ainsi que la nouvelle section intitulée "le Québec, le Canada, le monde "

Michel Lacroix [éd.], "Recensions ", Globe. Revue internationale d'études québécoises, vol. 6, $\mathrm{n}^{\circ} 2,2003$. 
qui permet d'aborder les relations internationales et d'inclure trois articles intéressants, l'un de Fernand Harvey sur les études québécoises dans le monde et leur positionnement stratégique par rapport aux études canadiennes, un autre de Ingo Kolboom qui propose une comparaison entre l'Allemagne et le Canada (justifiée par la nationalité de son auteur), suivi d'une présentation de l'Association internationale d'études québécoises (présidée par le même Ingo Kolboom).

On note un changement de ton dans l'avant-propos, puisque dans la version de 2001, l'accent était mis sur la question nationale et éventuellement sur l'hypothèse d'un troisième référendum et ce, dans le double contexte d'une économie florissante et de la grande réforme de Lucien Bouchard sur la vie politique municipale. L'avant-propos de cette septième édition revient sur le concept de société distincte, mais dans un contexte général de libre-échange et de mondialisation, tout en signalant le début d'une nouvelle ère politique suite à la démission de Lucien Bouchard et à l'arrivée au pouvoir de Bernard Landry.

Le plus grand intérêt de cet ouvrage est de constituer un indispensable instrument de référence sur les grandes tendances de fond de la société québécoise (au-delà des simples questions d'actualité), où se mêlent les regards croisés d'universitaires respectés et de journalistes avertis dans une pluridisciplinarité de très bon aloi. Le lecteur soucieux de mieux connaître le vrai visage du Québec trouvera ici une information rigoureuse ainsi que des interprétations politiques stimulantes présentées dans des textes de niveau universitaire agrémentés de tableaux et de cartes qui garantissent toujours une excellente vulgarisation. On saluera pour terminer la contribution quantitativement et qualitativement admirable de Simon Langlois qui, à lui seul, assure la production de 88 des 719 pages de texte et qui sensibilise notamment les lecteurs aux enjeux du défi démographique dans le Québec contemporain.

Jean-Michel Lacroix Université de Paris- Sorbonne 3 